PROCEEDINGS OF THE

AMERICAN MATHEMATICAL SOCIETY

Volume 133, Number 3, Pages 841-850

S 0002-9939(04)07657-9

Article electronically published on September 29, 2004

\title{
COMPONENTS OF LEVEL SETS OF UNIFORM CO-LIPSCHITZ FUNCTIONS ON THE PLANE
}

\author{
OLGA MALEVA \\ (Communicated by David Preiss)
}

\begin{abstract}
Consider a co-Lipschitz uniformly continuous function $f$ defined on the plane. Let $n(f)$ be the maximal number of components of its level set. In the present paper we settle a question of B. Randrianantoanina, concerning the dependence of $n(f)$ on the quantitative characteristics of the mapping. We prove that $n(f)$ is bounded from above by a simple function of the co-Lipschitz and the "weak Lipschitz" constants of $f$, and show that our estimate is sharp. We also prove additional properties of the level sets.
\end{abstract}

\section{INTRODUCTION}

Consider a mapping $f: U \rightarrow V$ between two normed spaces $U$ and $V$. The function

$$
\Omega_{f}(d)=\sup _{\|X-Y\|_{U} \leq d}\|f(X)-f(Y)\|_{V}
$$

is called the modulus of (uniform) continuity of $f$. The mapping $f$ is said to be uniformly continuous if $\Omega_{f}(d) \rightarrow 0$ as $d \downarrow 0$. In this case the modulus of continuity is a subadditive monotone continuous function.

One important class of uniformly continuous mappings is the class of Lipschitz mappings, i.e. those satisfying $\Omega_{f}(d) \leq L d$ for some positive $L$. The least such $L$ is called the Lipschitz constant of the mapping $f$. Note, however, that any uniformly continuous mapping is Lipschitz for large distances, because, as one may show, for any fixed $\varepsilon>0$,

$$
\|f(X)-f(Y)\|_{V} \leq \frac{\Omega_{f}(d)}{d}(1+\varepsilon)\|X-Y\|_{U}
$$

whenever $\|X-Y\|_{U} \geq \frac{d}{\varepsilon}$. Moreover, it is clear that $\frac{\Omega_{f}(d)}{d}$ is bounded for $d \geq d_{0}$ if $d_{0}$ is positive. We call the limit

$$
L_{f}^{*}=\lim _{d \rightarrow+\infty} \frac{\Omega_{f}(d)}{d}=\inf _{d>0} \frac{\Omega_{f}(d)}{d}<+\infty
$$

the weak Lipschitz constant of $f$. If $f$ is a Lipschitz mapping, $L_{f}^{*}$ does not exceed its Lipschitz constant.

Received by the editors November 5, 2002 and, in revised form, November 20, 2003.

2000 Mathematics Subject Classification. Primary 46B20.

The author was supported by the Israel Science Foundation.

(C)2004 American Mathematical Society Reverts to public domain 28 years from publication 
The definition of $\Omega_{f}$ implies that $f\left(B_{r}(x)\right) \subset B_{\Omega_{f}(r)}(f(x))$. In a similar way, co-uniformly continuous mappings are defined as those satisfying

$$
f\left(B_{r}(x)\right) \supset B_{\omega(r)}(f(x)), \quad r>0,
$$

for some function $\omega(r)>0$. A particular case is a co-Lipschitz mapping that satisfies

$$
f\left(B_{r}(x)\right) \supset B_{c r}(f(x)) .
$$

We call the best (the largest) such constant $c$ the co-Lipschitz constant of the mapping $f$. (Note that in some papers, in particular [JLPS, the co-Lipschitz constant means the inverse of our $c$.) Note that $c \leq L_{f}^{*}<\infty$ if the mapping $f$ is $c$-co-Lipschitz and uniformly continuous.

In the present paper we deal with uniformly continuous co-Lipschitz mappings from $\mathbb{R}^{2}$ to $\mathbb{R}$. Our subject is the structure of the level sets, i.e. the preimages of points under such mappings. Some basic examples related to this class of mappings and their point preimages were given in [JLPS, §5].

Our starting point will be the comprehensive description of the level sets of uniformly continuous co-Lipschitz functions $f$ defined on the plane given by Randrianantoanina in $\left[\mathbf{R}\right.$. By $\left[\underline{R}\right.$, Theorem 2.4] each level set $f^{-1}(t)$ has a finite number of components. According to [ $\mathbb{R}$, Theorem 4.11], every connected component $C$ of $f^{-1}(t)$ can be represented as

$$
C=C_{0} \cup \bigcup_{j=1}^{m} C_{j},
$$

where $C_{0}$ is a compact tree with $m$ leaves (end points), each $C_{j}$ is a ray, i.e. a closed unbounded set homeomorphic with $[0, \infty)$, the $C_{j}$ 's are disjoint, and $C_{j}$ has a unique common point with $C_{0}$, which is an end point of $C_{0}$ and the end point of $C_{j}$. Although it is intuitively clear what is an end point of a tree or of a ray, the topological definition of an end point can be found in [K Chapter VI, $\S 51]$.

The number of ends of such a connected component $C$ is the number of rays going to infinity (ends are not to be confused with end points), that is, $m$. The number of ends of $f^{-1}(t)$ is denoted by $\# e\left(f^{-1}(t)\right)$ and is the sum of the numbers of ends of all components of $f^{-1}(t)$.

Note that the number of ends of $W=f^{-1}(t)$ can also be defined in the following way (see [HR, Definition 1.18]). We say that $W$ has at least $k$ ends if there exists an open subset $V \subset W$ with compact closure $\bar{V}$ so that $W \backslash \bar{V}$ has at least $k$ unbounded components. We say that $W$ has exactly $k$ ends if $W$ has at least $k$ ends but not at least $k+1$ ends. If $W$ has exactly $k$ ends, we will write $\# e(W)=k$.

We see that if $W$ has exactly $k$ ends, then there exists a positive $d$ such that $W \backslash B_{d}$ has exactly $k$ unbounded components (by $B_{r}$ and $\bar{B}_{r}$ we denote, respectively, the open and the closed ball of radius $r$, centered at 0 ). Let us denote by $\Theta(W)$, say, the least such $d$ plus one. Then $W \backslash B_{d}$ has exactly $k$ unbounded components for any $d \geq \Theta(W)$. Denote the unbounded components of $W \backslash B_{\Theta(W)}$ by $C_{1}(W), \ldots, C_{k}(W)$ so that $\arg \left(C_{i}(W) \cap \bar{B}_{\Theta(W)}\right)<\arg \left(C_{i+1}(W) \cap \bar{B}_{\Theta(W)}\right)$ for all $i=1, \ldots, k-1$.

Theorem $5.1 \mathrm{in}[\mathrm{R}]$ states that for a $c$-co-Lipschitz uniformly continuous mapping $f: \mathbb{R}^{2} \rightarrow \mathbb{R}$, there exists a constant $n=n(f)$, such that for all $t \in \mathbb{R}$,

$$
\# e\left(f^{-1}(t)\right)=2 n
$$


and the maximal number of components of $f^{-1}(t)$ (over all $t \in \mathbb{R}$ ) is equal to $n$. This $n$ is bounded from above by a number $M=M\left(c, \Omega_{f}\right)$. In addition, if $\left\{C_{R, i}\right\}_{i=1}^{2 n}$ are unbounded components of $f^{-1}(t) \backslash B_{R}$, then for all $i \neq j$,

$$
\lim _{R \rightarrow \infty} \operatorname{dist}\left(C_{R, i}, C_{R, j}\right)=\infty .
$$

In the present paper we provide an explicit formula for $M\left(c, \Omega_{f}\right)$. We show that there exists a scale $0<\cdots<\rho_{2,1}^{(m)}<\cdots<\rho_{2,1}^{(1)}<1$ such that for any uniformly continuous $c$-co-Lipschitz mapping $f: \mathbb{R}^{2} \rightarrow \mathbb{R}$ the condition $c / L_{f}^{*}>\rho_{2,1}^{(m)}$ implies $n(f) \leq m$. In fact, such a scale is given by

$$
\rho_{2,1}^{(m)}=\sin \frac{\pi}{2(m+1)},
$$

so $M\left(c, \Omega_{f}\right)=\frac{\pi}{2 \arcsin \left(c / L_{f}^{*}\right)}$. We show that these scale values are precise, in the sense that there exist mappings $f_{m}: \mathbb{R}^{2} \rightarrow \mathbb{R}$ such that

$$
c_{f_{m}} / L_{f_{m}}^{*}=\rho_{2,1}^{(m-1)}=\sin (\pi / 2 m) \quad \text { and } \quad n\left(f_{m}\right)=m .
$$

The idea of a scale of this type was first suggested by the author in $\bar{M}$ for Lipschitz co-Lipschitz mappings from $\mathbb{R}^{2}$ to $\mathbb{R}^{2}$, where the ratio $c / L$ determines the sharp bound for the cardinality $n$ of point preimages. In fact, one may show that the following generalization of [M, Theorem 2] holds. For uniformly continuous c-co-Lipschitz mappings $f: \mathbb{R}^{2} \rightarrow \mathbb{R}^{2}$ one has $c / L_{f}^{*} \leq 1 / n$, where $n$ is the maximal cardinality of a point preimage. One may observe further analogies between the $\left(\mathbb{R}^{2}, \mathbb{R}^{2}\right)$ and $\left(\mathbb{R}^{2}, \mathbb{R}\right)$ situations, as mentioned in $[\underline{\mathbb{R}}$, Remark 5.2].

After constructing the scale for $n(f)$, we finish the paper by strengthening the property $\lim _{R \rightarrow \infty} \operatorname{dist}\left(C_{R, i}, C_{R, j}\right)=\infty$. We prove that not only the distance between the components $C_{R, i}$ and $C_{R, j}$ tends to infinity, but it is bounded from below by $\delta R$ for sufficiently large $R$ (see Proposition 1). This property implies, for example, that $f^{-1}(t)$ cannot contain a parabola.

The author thanks Beata Randrianantoanina for sending her preliminary versions of $[\underline{\mathrm{R}}$, for interesting discussions and very helpful comments on the subject, Joram Lindenstrauss for his valuable remarks on the preprint of this paper, Maxim Pratusevich for useful comments concerning subadditive functions, and Gideon Schechtman for all his help.

2 .

In this section we construct the scale for uniformly continuous co-Lipschitz mappings, as described in the Introduction. The main result is Theorem 11, and the sharpness of the bounds is demonstrated in the Remark after Theorem1. We start, however, with a few technical lemmas.

Lemma 1. If the mapping $f: \mathbb{R}^{2} \rightarrow \mathbb{R}$ is uniformly continuous and co-Lipschitz, $K$ is an unbounded component of $\left(\mathbb{R}^{2} \backslash B_{r}\right) \backslash f^{-1}(t)$ for some real $t$ and $r>0$, then $f(K)$ is unbounded.

Proof. Consider $r^{\prime}>\max \left\{r, \Theta\left(f^{-1}(t)\right)\right\}$. Then there exists an unbounded component $K^{\prime}$ of $\left(\mathbb{R}^{2} \backslash B_{r^{\prime}}\right) \backslash f^{-1}(t)$ such that $K^{\prime} \subset K$. Note that $\partial K^{\prime}$ is a union of two unbounded components $C_{1}$ and $C_{2}$ of $f^{-1}(t) \backslash B_{r^{\prime}}$ with a bounded curve $\Gamma$ whose ends are $C_{1} \cap \partial B_{r^{\prime}}$ and $C_{2} \cap \partial B_{r^{\prime}}$. 
By $[\underline{\mathrm{R}}$, Theorem $5.1,(1)]$ the distance between $C_{R, 1}$ and $C_{R, 2}$, where $C_{R, i}$ is the unbounded component of $C_{i} \backslash B_{R}$ for $i=1,2$, tends to infinity as $R$ tends to infinity. It follows that the set $K^{\prime} \backslash\left(U_{\rho}(\Gamma) \cup U_{\rho}\left(C_{1}\right) \cup U_{\rho}\left(C_{2}\right)\right)$ is nonempty for any $\rho>0$ (by $U_{s}(W)$ we denote the $s$ neighbourhood of the set $W$ ). Take any point $x$ in this set. Then the distance from $x$ to the boundary of $K^{\prime}$ is greater than $\rho$, that is, $K^{\prime}$ contains a ball of radius $\rho$. Therefore, since $f$ is co-Lipschitz, the image of $K^{\prime}$ under $f$ contains an interval of radius $c \rho$. This finishes the proof of the lemma, since $\rho$ can be arbitrarily large and $f(K)$ contains $f\left(K^{\prime}\right)$.

Corollary 1. Under the assumptions of Lemma $1, f(K)$ equals either $(t,+\infty)$ or $(-\infty, t)$.

Proof. First note that $f(x)-t$ has a constant sign inside $K$ (since the mapping $f$ is continuous and does not attain the value $t$ inside $K$ ). Assume, for example, that $f(x)>t$ for all $x \in K$. Then the fact that $f(K)$ is unbounded implies that $f(K)$ contains arbitrarily large real numbers. But this image is also connected and $t$ is its limit point, so $f(K)=(t,+\infty)$.

The next lemma is purely topological and will be used only in the proof of Lemma 3 We are going to use the following topological terminology. By an arc we mean a homeomorphic image of the segment $[0,1]$ (see $[\underline{K}, \S 47, \mathrm{~V}]$ ). We say that the arc $\gamma$ connects points $\omega_{1}$ and $\omega_{2}$ if $\omega_{i}, i=1,2$, are the end points of $\gamma$ (or, equivalently, $\omega_{1}=\gamma(0)$ and $\left.\omega_{2}=\gamma(1)\right)$. A ray means an unbounded homeomorphic image of $[0, \infty)$.

Lemma 2. Let $C_{1}$ and $C_{2}$ be two disjoint rays with end points $A_{1}$ and $A_{2}$ resp., and let $\Gamma$ be an arc with end points $A_{1}$ and $A_{2}$, not intersecting $C_{i}, i=1,2$, in other points. Let $K$ be one of the two open connected components of $\mathbb{R}^{2} \backslash\left(C_{1} \cup \Gamma \cup C_{2}\right)$ and $x \in K$. Then there exists $R=R(x, K)$ such that the following holds. If an arc $\gamma$ connects $A_{i}^{\prime} \in C_{i} \backslash B_{R}, i=1,2$, and $\gamma$ is such that $\gamma \backslash\left\{A_{1}^{\prime}, A_{2}^{\prime}\right\} \subset K \backslash B_{R}$, then every unbounded broken line inside $K$ containing $x$ intersects $\gamma$.

Proof. Note that $\partial K=C_{1} \cup \Gamma \cup C_{2}$. Let $L_{1} \neq L_{2}$ be two straight lines passing through $x$, each containing a point on $\partial K$. Let $I_{i}=\left[x, x_{i}\right] \subset L_{i}, i=1,2$, be two segments connecting $x$ with point $x_{i}$, on $\partial K$ so that $\left(x, x_{i}\right) \subset K$. Note that $\partial K \backslash\left\{x_{1}, x_{2}\right\}$ consists of two unbounded and one bounded component. Denote the closure of the bounded component by $\tau$.

Consider a simple closed curve $P_{1}=\tau \cup I_{1} \cup I_{2}$. By the Jordan Curve Theorem (see $\left[\mathrm{K}, \S 61\right.$, II, Theorem 1]) $P_{1}$ divides $\mathbb{R}^{2}$ into two connected components, which we will denote by $\operatorname{in}\left(P_{1}\right)$ and out $\left(P_{1}\right) ; \operatorname{in}\left(P_{1}\right)$ is bounded and out $\left(P_{1}\right)$ is unbounded.

Let $R(x, K)$ be such that $\operatorname{in}\left(\tau \cup I_{1} \cup I_{2}\right) \subset B_{R(x, K)}$. Let $\gamma$ be as described in the hypothesis of the lemma. Denote by $\tau_{i}, i=1,2$, the closures of the two bounded components of $(\partial K \backslash \tau) \backslash\left\{A_{1}^{\prime}, A_{2}^{\prime}\right\}$ so that $\tau_{i} \ni A_{i}^{\prime}$. Then the curve $P_{2}=\tau \cup \tau_{1} \cup \gamma \cup \tau_{2}$ is a simple closed curve. To prove the lemma, it is enough to demonstrate now that $\left(I_{1} \cup I_{2}\right) \backslash\left\{x_{1}, x_{2}\right\} \subset \operatorname{in}\left(P_{2}\right)$. Indeed, this would imply that $x \in\left(I_{1} \cup I_{2}\right) \backslash\left\{x_{1}, x_{2}\right\} \subset$ in $\left(P_{2}\right)$. So every unbounded broken line containing $x$ intersects $P_{2}$. Since the unbounded broken line is contained in $K$ and $\tau \cup \tau_{1} \cup \tau_{2} \subset \mathbb{R}^{2} \backslash K$, it can only intersect $P_{2}$ at a point belonging to $\gamma$.

In order to prove that $\left(I_{1} \cup I_{2}\right) \backslash\left\{x_{1}, x_{2}\right\} \subset \operatorname{in}\left(P_{2}\right)$, note that the points $x_{1}$ and $x_{2}$ are connected by three arcs: $\tau, I_{1} \cup I_{2}$ and $\tau_{1} \cup \gamma \cup \tau_{2}$. By the Theorem About The 
$\theta$-Curve ([K, $\S 61$, II, Theorem 2]), one of those arcs is contained in the bounded component defined by the two others. If such an arc is $I_{1} \cup I_{2}$, we are done.

Another option: $\left(\tau_{1} \cup \gamma \cup \tau_{2}\right) \backslash\left\{x_{1}, x_{2}\right\}$ is a subset of $\operatorname{in}\left(\left(I_{1} \cup I_{2}\right) \cup \tau\right)$. This is impossible since the latter is inside the ball $B_{R(x, K)}$, which does not intersect $\gamma$.

Third option: $\tau \backslash\left\{x_{1}, x_{2}\right\} \subset \operatorname{in}\left(\left(I_{1} \cup I_{2}\right) \cup\left(\tau_{1} \cup \gamma \cup \tau_{2}\right)\right)$. In order to refute this, we will show now that $\operatorname{in}\left(\left(I_{1} \cup I_{2}\right) \cup\left(\tau_{1} \cup \gamma \cup \tau_{2}\right)\right) \subset K$, and since $\tau \subset \mathbb{R}^{2} \backslash K$, the third option is also impossible.

Let $P_{3}=I_{1} \cup I_{2} \cup \tau_{1} \cup \gamma \cup \tau_{2}$. Clearly, $P_{3}$ is a subset of the closure $\bar{K}$. We would like to show that $\operatorname{in}\left(P_{3}\right) \subset K$. Note that $\mathbb{R}^{2} \backslash P_{3}$ contains an unbounded component $\widetilde{C}_{1}$ of $C_{1} \backslash\left\{A_{1}^{\prime}\right\}$. Therefore, $\widetilde{C}_{1} \subset \operatorname{out}\left(P_{3}\right)$. Since out $\left(P_{3}\right)$ is open, it contains a small neighbourhood of some point $M \in \widetilde{C}_{1}$. Since $C_{1}$ lies in the boundary of $K$, every neighbourhood of $M$ contains points of $K^{\prime}=\left(\mathbb{R}^{2} \backslash K\right) \backslash \partial K\left(K^{\prime}\right.$ and $K$ are the two components of $\left.\mathbb{R}^{2} \backslash \partial K\right)$. Thus, out $\left(P_{3}\right)$ contains points from $K^{\prime}$. Since $K^{\prime} \cap P_{3}=\emptyset$ and $K^{\prime}$ is connected, we conclude that $K^{\prime} \subset \operatorname{out}\left(P_{3}\right)$. Therefore, $\operatorname{in}\left(P_{3}\right) \subset \mathbb{R}^{2} \backslash K^{\prime}=\bar{K}$. But the set $\operatorname{in}\left(P_{3}\right)$ is open, so in $\left(P_{3}\right) \subset K$.

Lemma 3. Let $f: \mathbb{R}^{2} \rightarrow \mathbb{R}$ be a uniformly continuous, c-co-Lipschitz mapping and $K$ be an unbounded component of $\left(\mathbb{R}^{2} \backslash B_{d}\right) \backslash f^{-1}(t)$ for some real $t$ and $d>$ $\Theta\left(f^{-1}(t)\right)$. Then for any $\widetilde{c}<c$ there exists a positive number $R(\widetilde{c})$ with the following property.

Let $\gamma$ be any arc whose end points $\gamma(0)$ and $\gamma(1)$ belong to different unbounded components of $f^{-1}(t) \backslash B_{d}$ and such that $\gamma(s) \in K \backslash B_{R(\widetilde{c})}$ for all $s \in(0,1)$. Then one can find a point $y \in \gamma$, such that $|f(y)-t|>\widetilde{c}\|y\|$.

Proof. Let $\varepsilon=\Omega_{f}^{-1}(1)$. Then $|f(X)-f(Y)| \leq 1$ if $\|X-Y\| \leq \varepsilon$. Without loss of generality, we may assume that $t=0$. Assume also that $f$ is positive inside $K$. Then by Corollary $f(K)=(0,+\infty)$, and thus we may take $x_{0} \in K$ such that

$$
f\left(x_{0}\right)>1+\max _{x \in \bar{B}_{d}}|f(x)| .
$$

Consider a ball $D_{0}$ of radius $\varepsilon$ centered at $x_{0}$. Since $f\left(x_{0}\right)>1$, the closure $\bar{D}_{0}$ contains no point whose image is 0 . Moreover, $\bar{D}_{0}$ does not intersect $B_{d}$, since $f\left(x_{0}\right)>1+f(x)$ for any $x \in B_{d}$. Thus $\bar{D}_{0}$ is inside $K$. But $f\left(\bar{D}_{0}\right)$ contains the segment $\left[f\left(x_{0}\right)-c \varepsilon, f\left(x_{0}\right)+c \varepsilon\right]$. In particular, there exists $x_{1} \in \bar{D}_{0} \subset K$ such that $f\left(x_{1}\right)=f\left(x_{0}\right)+c \varepsilon$.

Now we are going to construct a sequence of points $x_{l} \in K$ in the following way. Suppose $x_{l} \in K$ is already constructed, and $f\left(x_{l}\right)>f\left(x_{0}\right)$ (in the beginning, this holds for $\left.x_{1}\right)$. Then we define $D_{l}=B\left(x_{l}, \varepsilon\right)$, and notice that $\bar{D}_{l}$ is inside $K$. By coLipschitzness, $f\left(\bar{D}_{l}\right)$ contains $\left[f\left(x_{l}\right)-c \varepsilon, f\left(x_{l}\right)+c \varepsilon\right]$, so there exists $x_{l+1} \in \bar{D}_{l} \subset K$ such that $f\left(x_{l+1}\right)=f\left(x_{l}\right)+c \varepsilon$.

Note that this procedure implies the following equality:

$$
f\left(x_{l}\right)=f\left(x_{0}\right)+l c \varepsilon .
$$

In particular, $f\left(x_{l}\right) \rightarrow+\infty$, and thus $x_{l} \rightarrow \infty$. Let $l_{0}$ be the first index such that $l_{0}>\frac{\widetilde{c}\left\|x_{0}\right\|}{(c-\widetilde{c} \varepsilon}$. Let $R(\widetilde{c})$ be $R\left(x_{l_{0}}, K\right)$ from Lemma 2

Consider any arc $\gamma$ with properties as in the assumptions of the lemma. Since the broken line connecting $x_{l_{0}}, x_{l_{0}+1}, \ldots$ is entirely inside $K$ and is unbounded, there is a point of intersection of this broken line with $\gamma$. Consider $l_{1} \geq l_{0}$ such that the segment $\left[x_{l_{1}}, x_{l_{1}+1}\right]$ intersects $\gamma$, and let $y$ be a point of intersection: $y \in$ 
$\left[x_{l_{1}}, x_{l_{1}+1}\right] \cap \gamma$. Then $\|y\| \leq \max \left\{\left\|x_{l_{1}}\right\|,\left\|x_{l_{1}+1}\right\|\right\} \leq\left\|x_{0}\right\|+\left(l_{1}+1\right) \varepsilon$ and $\left\|y-x_{l_{1}+1}\right\| \leq$ $\varepsilon$. It follows that

$$
\begin{aligned}
f(y) & \geq f\left(x_{l_{1}+1}\right)-1=f\left(x_{0}\right)+\left(l_{1}+1\right) c \varepsilon-1>\left(l_{1}+1\right) c \varepsilon \\
& =\widetilde{c}\left(l_{1}+1\right) \varepsilon+\left(l_{1}+1\right)(c-\widetilde{c}) \varepsilon>\widetilde{c}\left(l_{1}+1\right) \varepsilon+\widetilde{c}\left\|x_{0}\right\|=\widetilde{c}\left(\left(l_{1}+1\right) \varepsilon+\left\|x_{0}\right\|\right),
\end{aligned}
$$

so we conclude that

$$
|f(y)|=f(y)>\widetilde{c}\|y\| .
$$

Corollary 2. Let $f: \mathbb{R}^{2} \rightarrow \mathbb{R}$ be a uniformly continuous, c-co-Lipschitz mapping and $K$ be an unbounded component of $\left(\mathbb{R}^{2} \backslash B_{d}\right) \backslash f^{-1}(t)$ for some real $t$ and $d>$ $\Theta\left(f^{-1}(t)\right)$. Then for any $\widetilde{c}<c$ there exists a positive number $R(\widetilde{c})$ with the following property. If $r>R(\widetilde{c})$, then one can find a point $y$ in $K$, of norm $r$, such that $|f(y)-t|>\widetilde{c}\|y\|$.

Proof. Let $C_{1}$ and $C_{2}$ be the two unbounded components of $f^{-1}(t) \backslash B_{d}$ that lie in the boundary of $K$. Take $R(\widetilde{c})$ provided by Lemma 3 and enlarge it, if necessary, so that any circle of radius $r>R(\widetilde{c})$ does not intersect the bounded set $\Gamma=$ $\partial K \backslash\left(C_{1} \cup C_{2}\right)$. Then for any $r>R(\widetilde{c})$ the intersection of $K$ and $\partial B_{r}$ is a union of disjoint open arcs whose endpoints lie in $\partial K \cap \partial B_{r} \subset C_{1} \cup C_{2}$.

An argument below shows that at least one of these disjoint open arcs, say $\gamma$, has the property that one of the endpoints of $\gamma$ is in $C_{1}$, and the other is in $C_{2}$. To finish the proof of the corollary, it remains to apply Lemma 3 to $\gamma$.

Let us show how to find the open arc $\gamma$. By [R, Corollary 5.12] we may assume $d$ to be such that the distance $\epsilon=\operatorname{dist}\left(C_{1}, C_{2}\right)$ is positive. Fix any $r>R(\widetilde{c})$ so that $\Gamma \subset B_{r}$. Denote by $U_{s}(W)$ the $s$-neighbourhood of a set $W$. The set $\Gamma=\partial K \backslash\left(C_{1} \cup C_{2}\right)$ is not covered by $U_{\epsilon / 3}\left(C_{1}\right) \cup U_{\epsilon / 3}\left(C_{2}\right)$ (otherwise, since $\Gamma$ is connected, there would be a point on $\Gamma$ with distances at most $\epsilon / 3$ both from $C_{1}$ and $C_{2}$, which is impossible). Therefore, there exists a point $x \in \Gamma$ such that $\operatorname{dist}\left(x, C_{i}\right)>\epsilon / 3, i=1,2$.

Note that the intersection $K \cap B_{\epsilon / 6}(x)$ is nonempty, since $x$ is a point on the boundary of $K$. Take any point $y$ in this intersection. Then the distance dist $(y, \partial K)$ does not exceed $\epsilon / 6$. Consider $z \in \partial K$ such that

$$
\|y-z\|=\operatorname{dist}(y, \partial K) \leq \epsilon / 6 .
$$

Then the half-open interval $(z, y]$ is inside $K$ and $\|x-z\|<\epsilon / 3$, that is, $z \notin C_{1} \cup C_{2}$.

Let us construct an unbounded broken line $\mathcal{L}_{y}$ inside $K$, whose vertices moreover tend to infinity, which starts at $y$. We may do it, connecting $y$ with a point $y^{\prime} \in K$, such that $\left|f\left(y^{\prime}\right)-t\right|>1+\max _{w \in \bar{B}_{d}}|f(w)-t|$ and then using the procedure described in the proof of Lemma 3. Let $\mathcal{L}=[z, y] \cup \mathcal{L}_{y}$.

Without loss of generality, we may assume that the vertices of $\mathcal{L}$ do not lie on the circle of radius $r$, centered at zero. First of all, there may be only finitely many such vertices (recall that norms of vertices of $\mathcal{L}$ tend to infinity). Furthermore, since $K$ is open, it contains every vertex of $\mathcal{L}$ (except $z$ ) together with a small open neighbourhood. So if a vertex lies on $\partial B_{r}$, then we may replace a small part of $\mathcal{L}$ inside this neighbourhood by a segment so that there is no longer a vertex on the circle.

This broken line $\mathcal{L}$ starts inside $B_{r}$, since $z \in \Gamma \subset B_{r}$, goes to infinity and has no vertices on the circle $\partial B_{r}$. Therefore, $\mathcal{L}$ has an odd number of intersections with $\partial B_{r}$. Since $\mathcal{L} \backslash\{z\} \subset K$, all its intersections with the circle of radius $r$ lie in 
$K \cap \partial B_{r}$, which is a union of open disjoint arcs, among which we would like to find $\gamma$ such that its endpoints belong to $C_{1}$ and $C_{2}$.

Let $\gamma$ be such an open arc that has an odd number of intersections with $\mathcal{L}$. Assume towards a contradiction that both endpoints of $\gamma$ belong to, say, $C_{1}$. Then consider a path $\tau \subset C_{1}$ connecting those two points. Note that $\tau \cap \gamma=\emptyset$, since $\gamma \subset K$, and $\tau \cap K \subset C_{1} \cap K=\emptyset$. Then, since the endpoints of $\gamma$ and $\tau$ coincide, the union $\tau \cup \gamma$ is a simple closed curve. Since $\mathcal{L} \backslash\{z\} \subset K$ and $z \notin C_{1}$, we conclude $\mathcal{L} \cap \tau=\emptyset$. This means that the number of intersections between the broken line $\mathcal{L}$ and the simple closed curve $\tau \cup \gamma$ is odd. Since the broken line goes to infinity, by the Jordan Curve Theorem $(\underline{\mathrm{K}}, \S 61$, II, Theorem 1]) its startpoint $z$ is inside the bounded component of $\mathbb{R}^{2} \backslash(\tau \cup \gamma)$.

Consider one of the two components of $\partial K \backslash\{z\}$ that contains $C_{2}$. This curve does not intersect $C_{1}$, and so does not intersect $\tau$; this curve does not intersect $\gamma$, since $\gamma \subset K$. With all this, the curve connects the point $z$, which is inside the bounded component of $\mathbb{R}^{2} \backslash(\tau \cup \gamma)$, with infinity. This is a contradiction.

Hence both endpoints of the arc $\gamma$ cannot lie in $C_{1}$. An identical argument shows they cannot both lie in $C_{2}$. Thus, one of the endpoints of $\gamma$ is in $C_{1}$ and the other is in $C_{2}$.

Recall that by $C_{1}\left(f^{-1}(t)\right), \ldots, C_{2 n}\left(f^{-1}(t)\right)$ we denote the unbounded components of $f^{-1}(t) \backslash B_{\Theta\left(f^{-1}(t)\right)}$ so that

$$
\arg \left(C_{i}\left(f^{-1}(t)\right) \cap \bar{B}_{\Theta\left(f^{-1}(t)\right)}\right)<\arg \left(C_{i+1}\left(f^{-1}(t)\right) \cap \bar{B}_{\Theta\left(f^{-1}(t)\right)}\right)
$$

for all $i=1, \ldots, 2 n-1$.

Lemma 4. Let $f: \mathbb{R}^{2} \rightarrow \mathbb{R}$ be a co-Lipschitz and uniformly continuous mapping with modulus of continuity $\Omega_{f}(r)$. Suppose $L_{f}^{*}<1$. Take $t \in \mathbb{R}$ and denote $2 n=$ $\# e\left(f^{-1}(t)\right)$. Then for any sufficiently large $r$,

$$
\min _{i} \max _{x \in \Gamma_{i}(r)}|f(x)-t| \leq r \sin (\pi / 2 n),
$$

where $\Gamma_{i}(r)$ is the arc of the circle of radius $r$ defined in the following way. For each $r>\Theta\left(f^{-1}(t)\right)$ we fix $2 n$ points $A_{i}(r) \in C_{i}\left(f^{-1}(t)\right)$ on the circle of radius $r$, and by $\Gamma_{i}(r), i=1, \ldots, 2 n$ denote the closed arc going counter-clockwise from $A_{i}(r)$ to $A_{i+1}(r)$ (here $A_{2 n+1}(r)$ is identified with $A_{1}(r)$ ).

Proof. Assume there are arbitrarily large $r$ 's such that the inequality does not hold. Note that $|f(x)-t| \leq \Omega_{f}\left(\operatorname{dist}\left(x, f^{-1}(t)\right)\right)$ for each $x$. So we have that for arbitrarily large $r$, for each $i$,

$$
\max _{x \in \Gamma_{i}(r)} \Omega_{f}\left(\operatorname{dist}\left(x, f^{-1}(t)\right)\right)>r \sin (\pi / 2 n) .
$$

Since $L_{f}^{*}<1$, there exists $R_{1}$ such that $\Omega_{f}(r)<r$ for $r \geq R_{1}$. If $r$ is so large that $r \sin (\pi / 2 n)>R_{1}$, then $r \sin (\pi / 2 n)>\Omega_{f}(r \sin (\pi / 2 n))$, i.e. for arbitrarily large $r$ and for each $i$,

$$
\max _{x \in \Gamma_{i}(r)} \Omega_{f}\left(\operatorname{dist}\left(x, f^{-1}(t)\right)\right)>\Omega_{f}(r \sin (\pi / 2 n)) .
$$

Since $\Omega_{f}(\cdot)$ is an increasing function, it follows that

$$
\max _{x \in \Gamma_{i}(r)} \operatorname{dist}\left(x, f^{-1}(t)\right)>r \sin (\pi / 2 n) .
$$


The latter means that for each $i$ there exists $x_{i} \in \Gamma_{i}(r)$ such that the closed ball $\bar{D}_{i}$ of radius $r \sin (\pi / 2 n)$ centered at $x_{i}$ does not intersect $f^{-1}(t)$. This implies that $\bar{D}_{i}$ is contained in the same component of $\mathbb{R}^{2} \backslash f^{-1}(t)$ as $x_{i}$.

Consider the case $n>1$. Since $r$ can be chosen arbitrarily large, we may assume that $r-r \sin (\pi / 2 n)>\Theta\left(f^{-1}(t)\right)$, so we conclude that $\bar{D}_{i}$ is contained in the same component of $\left(\mathbb{R}^{2} \backslash B_{\Theta\left(f^{-1}(t)\right)}\right) \backslash f^{-1}(t)$ as $x_{i}$. In particular, $\bar{D}_{i} \cap \bar{D}_{j}=\emptyset$ for any $i \neq j$.

In the case $n=1, x_{1}$ and $x_{2}$ (and therefore $\bar{D}_{1}$ and $\bar{D}_{2}$ ) will be in two disjoint components of $\mathbb{R}^{2} \backslash f^{-1}(t)$, so again $\bar{D}_{1} \cap \bar{D}_{2}=\emptyset$.

On the other hand, it is impossible to find $m \geq 2$ points on a circle of radius $r$ so that the closed balls of radius $r \sin (\pi / m)$ centered at those points will be disjoint. (Otherwise, the polygon with vertices at those points would have perimeter greater than $2 m r \sin (\pi / m)$; but the latter is the perimeter of the regular $m$-gon, which is maximal among all perimeters of $m$-gons inscribed in the circle of radius $r$.) This simple geometric observation finishes the proof of the lemma.

Theorem 1. Let $f: \mathbb{R}^{2} \rightarrow \mathbb{R}$ be a c-co-Lipschitz and uniformly continuous mapping with modulus of continuity $\Omega_{f}(r)$ and $n$ be the maximal number of components of the level sets $f^{-1}(t)$. Then $c / L_{f}^{*} \leq \sin (\pi / 2 n)$.

Proof. Assume $c / L_{f}^{*}>\sin (\pi / 2 n)$. Then by rescaling we may assume that $L_{f}^{*}<1$ and $c>\sin (\pi / 2 n)$. Let $\widetilde{c}=\sin (\pi / 2 n)$; then $c>\widetilde{c}$. For each unbounded component $K_{i}, i=1, \ldots, 2 n$, of $\left(\mathbb{R}^{2} \backslash B_{\Theta\left(f^{-1}(0)\right)}\right) \backslash f^{-1}(0)$ we may find $R_{i}(\widetilde{c})$ from Corollary 2 Then for all $r>\max _{i} R_{i}(\widetilde{c})$ there exist $2 n$ points $y_{i} \in K_{i}$ such that $\left\|y_{i}\right\|=r$ and $\left|f\left(y_{i}\right)\right|>\widetilde{c}\left\|y_{i}\right\|=\widetilde{c} r$. Therefore for all $r>\max _{i} R_{i}(\widetilde{c})$,

$$
\min _{i} \max _{x \in \Gamma_{i}(r)}|f(x)|>\widetilde{c} r=r \sin (\pi / 2 n) .
$$

This contradicts Lemma 4.

Remark. Note that the estimate given in Theorem 1 is sharp. It is attained at the following mapping $f$. In Figure 1 denote by $f(x)$ the distance from $x$ to the union of the $n$ solid lines, multiplied, in each component of the complement of the solid lines, by the sign indicated. Then $f$ is 1-weakly Lipschitz (and actually 1-Lipschitz), $\sin (\pi / 2 n)$ co-Lipschitz, and $2 n=\# e\left(f^{-1}(0)\right)$.

\section{3.}

We prove here that the distance between any two different unbounded components of $f^{-1}(t) \backslash B_{R}$ is bounded from below by $\delta R$ for sufficiently large $R$.

Proposition 1. Let $f: \mathbb{R}^{2} \rightarrow \mathbb{R}$ be a c-co-Lipschitz and uniformly continuous mapping with modulus of continuity $\Omega_{f}(r)$. Then there exists a positive $\delta>0$ that depends only on $c$ and $\Omega_{f}$ with the following property. For any $t$ there exists $\sigma=\sigma(t)>0$ such that $\operatorname{dist}\left(C_{i, R}, C_{j, R}\right) \geq \delta R$ for $R>\sigma$ and $i \neq j$. Here $C_{i, R}$ is the unbounded component of $C_{i}\left(f^{-1}(t)\right) \backslash B_{R}$.

Proof. Fix any real $t$. Let $2 n=\# e\left(f^{-1}(t)\right)$, and let $C_{i}=C_{i}\left(f^{-1}(t)\right)$ for all $i=1, \ldots, 2 n$. 


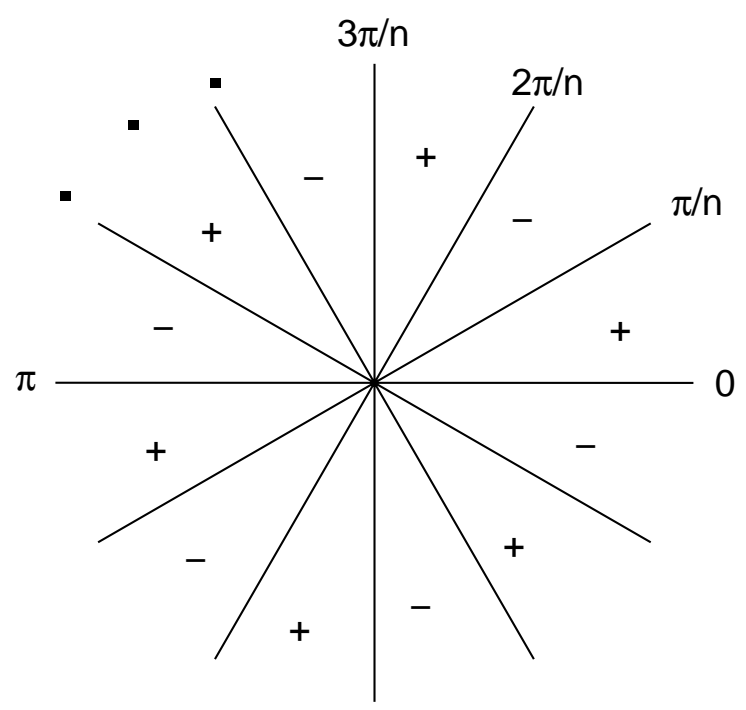

Figure 1 . The angle between any two adjacent rays is $\pi / n$.

By rescaling we may assume that $L_{f}^{*}<1$. Fix $d_{0}$ such that $\Omega_{f}(d)<d$ for all $d \geq d_{0}$. Choose any $\widetilde{c}<c$, and let $\delta=\widetilde{c} \sqrt{2}$. Consider

$$
\sigma=\left(\sqrt{1-(\delta / 2)^{2}}\right)^{-1} \max \left\{\frac{d_{0} \sqrt{2}}{\delta}, \max _{1 \leq i<j \leq 2 n} R_{i, j}(\widetilde{c}), \Theta\left(f^{-1}(t)\right)\right\},
$$

where $R_{i, j}(\widetilde{c})$ is the maximum of two constants from Lemma 3 for two components of $S_{i, j}=\left(\mathbb{R}^{2} \backslash B_{\Theta\left(f^{-1}(t)\right)}\right) \backslash\left(C_{i} \cup C_{j}\right)$.

Consider any $i \neq j$, and assume that $\operatorname{dist}\left(C_{i, R}, C_{j, R}\right)<\delta R$ for $R>\sigma$. This means that there exist $x_{1} \in C_{i, R}$ and $x_{2} \in C_{j, R}$ such that $\left\|x_{1}-x_{2}\right\|=\operatorname{dist}\left(C_{i, R}, C_{j, R}\right)$ $<\delta R$. Since $\left\|x_{1}\right\|,\left\|x_{2}\right\| \geq R$ and $\left\|x_{1}-x_{2}\right\|<\delta R$, for any point $p \in\left[x_{1}, x_{2}\right]$ one has $\|p\| \geq R \sqrt{1-(\delta / 2)^{2}}>\sigma \sqrt{1-(\delta / 2)^{2}} \geq \Theta\left(f^{-1}(t)\right)$. Then the open interval $\left(x_{1}, x_{2}\right)$ lies inside one of the two components of $S_{i, j}$. Moreover, the norm of each point belonging to $\left[x_{1}, x_{2}\right]$ is at least $R_{i, j}(\widetilde{c})$; thus $\left[x_{1}, x_{2}\right] \cap B_{R_{i, j}(\widetilde{c})}=\emptyset$.

Then by Lemma 3 applied to $\gamma=\left[x_{1}, x_{2}\right]$, there exists $y \in\left[x_{1}, x_{2}\right]$ such that $|f(y)-t|>\widetilde{c}\|y\|$. On the other hand, if both $\left\|y-x_{1}\right\|$ and $\left\|y-x_{2}\right\|$ are greater than $d_{0}$, then

$$
\begin{aligned}
& |f(y)-t|=\left|f(y)-f\left(x_{1}\right)\right|=\left|f(y)-f\left(x_{2}\right)\right| \\
& \leq \min \left\{\Omega_{f}\left(\left\|y-x_{1}\right\|\right), \Omega_{f}\left(\left\|y-x_{2}\right\|\right)\right\} \leq \min \left\{\left\|y-x_{1}\right\|,\left\|y-x_{2}\right\|\right\} \\
& \leq \delta R / 2 \leq \frac{\delta}{2 \sqrt{1-(\delta / 2)^{2}}}\|y\| \leq \delta\|y\| / \sqrt{2}=\widetilde{c}\|y\| .
\end{aligned}
$$

If, say, $\left\|y-x_{1}\right\| \leq d_{0}$, then

$$
\begin{aligned}
& |f(y)-t| \leq \Omega_{f}\left(d_{0}\right)<d_{0} \leq \frac{\delta \sigma \sqrt{1-(\delta / 2)^{2}}}{\sqrt{2}}<\frac{\delta R \sqrt{1-(\delta / 2)^{2}}}{\sqrt{2}} \\
& \leq \delta\|y\| / \sqrt{2}=\widetilde{c}\|y\| .
\end{aligned}
$$


In either case we get a contradiction. So the assumption that the distance between $C_{i, R}$ and $C_{j, R}$ is less than $\delta R$ was wrong.

Corollary 3. If $f: \mathbb{R}^{2} \rightarrow \mathbb{R}$ is a uniformly continuous co-Lipschitz mapping, then its level set cannot contain a whole parabola.

\section{REFERENCES}

[HR] B. Hughes, A. Ranicki, Ends of complexes, Cambridge University Press, Cambridge, 1996. MR1410261 (98f:57039)

[JLPS] W. B. Johnson, J. Lindenstrauss, D. Preiss, and G. Schechtman, Uniform quotient mappings of the plane, Michigan Math. J. 47 (2000), 15-31. MR.1755254(2001a:54037)

[K] K. Kuratowski, Topology, Vol. II, Academic Press, New York, 1968. MR.0259835 (41:4467)

$[\mathrm{M}] \quad$ O. Maleva, Lipschitz quotient mappings with good ratio of constants, Mathematika 49 (2002), no. 1-2, 159-165. MR2059051

[R] B. Randrianantoanina On the structure of level sets of uniform and Lipschitz quotient mappings from $\mathbb{R}^{n}$ to $\mathbb{R}$, Geom. Funct. Anal. 13 (2003), 1329-1358. MR2033841

Department of Mathematics, The Weizmann Institute of Science, Rehovot 76100, ISRAEL

Current address: Department of Mathematics, University College London, Gower Street, London, WC1E 6BT, United Kingdom

E-mail address: olga@math.ucl.ac.uk 